Opinion

\title{
Microbial Metagenomics Reveals Climate-relevant Subsurface Biogeochemical Processes
}

\author{
Philip E. Long ${ }^{1}{ }^{*}$ Kenneth H. Williams $^{1}$, Susan S. Hubbard ${ }^{1}$ and \\ Jillian F. Banfield ${ }^{1,2}$ \\ ${ }^{1}$ Earth Science Division, Lawrence Berkeley National Laboratory, Berkeley, CA 94720 \\ USA \\ ${ }^{2}$ Department of Earth and Planetary Science, University of California, Berkeley, \\ Berkeley, CA 94720 USA \\ *Correspondence: pelong@lbl.gov (P.E. Long).
}

\begin{abstract}
Microorganisms play key roles in terrestrial system processes, including the turnover of natural organic carbon, such as leaf litter and woody debris that accumulate in soils and subsurface sediments. What has emerged from a series of recent DNA sequencing-based studies is recognition of the enormous variety of little known and previously unknown microorganisms that mediate recycling of these vast stores of buried carbon in subsoil compartments of the terrestrial system. More importantly, the genome resolution achieved in these studies has enabled association of specific members of these microbial communities with carbon compound transformations and other linked biogeochemical processes such as the nitrogen cycle that can impact the quality of groundwater, surface water, and atmospheric trace gas concentrations. The emerging view also emphasizes the importance of organism interactions through exchange of metabolic byproducts (e.g., within the carbon, nitrogen, and sulfur cycles) and via symbioses since many novel organisms exhibit restricted metabolic capabilities and an associated extremely small cell size. New, genome-resolved information reshapes our view of subsurface microbial communities and provides critical new inputs for advanced reactive transport models. These inputs are needed for accurate prediction of feedbacks in watershed biogeochemical functioning and their influence on the climate via the fluxes of greenhouse gases, $\mathrm{CO}_{2}, \mathrm{CH}_{4}$, and $\mathrm{N}_{2} \mathrm{O}$.
\end{abstract}

Keywords: Metagenome, subsurface biogeochemistry, reaction pathway, greenhouse gases

\section{The Global Importance of Subsurface Microbial Communities}

We stand on the cusp of a much deeper grasp of subsurface microbes as agents of biogeochemistry than has ever been achieved before. Understanding of subsurface microbial communities is important at this juncture because the subsurface remains poorly represented in current models of biogeochemical processes, while DNA sequencing and mass spectrometric capabilities now position us to explore this topic comprehensively via metagenomics, metatranscriptomics and metaproteomics[1-6]. By "subsurface" we mean that part of the earth below the surface of the terrestrial landscape. As practical matter, this paper is focused on the shallow subsurface, which we arbitrarily 
define as $<10 \mathrm{~m}$ below the terrestrial surface. The practical and scientific implications of understanding microbial community structure and interactions among species at a mechanistic level, including species present at $<0.1 \%$ of the community, in this environment are enormous[7,8]. These include greatly improved ability to (i) estimate greenhouse gas fluxes to and from the subsurface via modeling of microbially driven $\mathrm{CO}_{2}$ fixation and degradation of organic matter, (ii) environmental management of mining impacts from toxic metals via a complete understanding of microbial metal redox cycles, and (iii) improved design of waste handling from animal confinements via the ability to accurately model nitrogen cycling in the subsurface. A key scientific impact is that we now 'see' all of the species in an environmental sample, including those from previously unknown lineages, and this informs major revisions of the tree of life[9]. Newly acquired data that reveal what specific microbes can and cannot do also impact our understanding of relationships among microbial species. Other benefits are perhaps less predictable but no less real, for example the discovery of new biocatalysts from genes in the vast array of new microbial genomes.

We posit that recently discovered microorganisms from the terrestrial subsurface, now known almost exclusively from metagenomics, play key roles in subsurface biogeochemical reactions that control fluxes of climate-relevant gases $\left(\mathrm{CO}_{2}, \mathrm{CH}_{4}, \mathrm{~N}_{2} \mathrm{O}\right.$, http://cdiac.ornl.gov/pns/current_ghg.html) including breakdown of buried organic carbon (Figure 1). Moreover, to understand the role of subsurface microbial communities in environmental processes it is necessary to genomically analyze a representative set of subsurface environments in a thorough and comprehensive manner such that complete or nearly complete genomes for nearly all community members are obtained. Such genomeresolved information, regardless of whether it uncovers novel organisms or not, is key to understanding the metabolic reactions possible in the subsurface and is a prerequisite for interpreting metatranscriptomic and metaproteomic datasets that provide information about the actual metabolic functions occurring at any time or under specific conditions [10]. In combination, these data represent key microbiologic inputs needed for effective modeling of biogeochemical fluxes. While discussion of modeling of such fluxes is beyond the scope of this paper, it is important to keep that goal in mind, because ultimately greenhouse gas fluxes from the terrestrial subsurface need to be predicted under conditions of climate change.

\section{New Insights from Genomic Data on Subsurface Ecosystems}

Living organisms are classified into three domains Bacteria, Archaea, and Eukarya, distinguished by their genetic makeup, cellular structure, and metabolic traits[11]. Eukaryotes include complex life forms such as mammals, birds, and fish. Archaea and bacteria dominate life in the subsurface, because they fit into soil, sediment, and rock pores and have diverse metabolic capabilities that operate in the subsurface under a wide range of oxygen concentrations. Subsurface microbial communities both take advantage of, and create biogeochemical gradients that influence fluxes of key biogeochemical species from the subsurface to rivers and the atmosphere. A wide range of metabolisms occur in the subsurface, including heterotrophy (particularly in soils or sediments with buried organic matter), and chemolithoautotrophy, which enables fixation of carbon using 
energy from redox transitions involving variable-redox elements in the Earth's crust (e.g. $[3,12,13])$.

The number of species of single-celled organisms in the Earth is unknown, but it is clear that there are a very large number of rare microorganisms that constitute an important part of the Earth's ecology, catalyzing biogeochemical reactions and serving as a microbial 'bank' from which new consortia can be 'seeded' as conditions change[14]. Only a minute fraction of these species have been grown in the single organism cultures that have traditionally been used for microbial characterization[15]. It may not be feasible to isolate most species for laboratory study because they require unknown inputs from other organisms or their natural environments. To understand how these organisms function, both individually and collectively, it is therefore necessary to use methods independent of isolation and culturing[16]. DNA sequencing-based means to do this have become available only relatively recently, and with decreasing cost and increasing speed, the approach can now be applied to even the most complex microbial ecosystems. The methods rely upon new bioinformatics approaches that can make use of small snippets of DNA, assembling them into genome fragments and then into near-complete or even complete genomes from which organism functions can be predicted[17, 18].

Recent research on samples of sediment and groundwater from a research site near the town of Rife, Colorado, USA has used massive amounts of genomic (DNA sequence) data ( 10 gigabases, $\mathrm{Gb}$, where a $\mathrm{Gb}=10^{9}$ bases, per sample; a few terabases total, $\mathrm{Tb}=$ $10^{12}$ bases) to reveal previously unrecognized metabolic potential and diversity in the shallow subsurface. A novel aspect of this work is the genome-centric approach, which has yielded thousands of genomes including the first complete archaeal genomes from metagenomic data. This approach has enabled an in-depth exploration of major segments of both the bacterial and archaeal trees of life, about which little was known previously (Figure 2)[9]. Results from applying these methods to samples of groundwater and sediment yield a tally of the microorganisms present, including species that comprise less than 0.01 percent of the communities[19]. Moreover, the biogeochemical reactions catalyzed by each organism can be ascertained, as well as how products of one organism may provide resources for another. The dominant reactions can then be incorporated into reaction networks to understand the life strategies of the microorganisms and the overall functioning of the community[20]. The reaction networks in turn are used in quantitative models that calculate subsurface properties, such as the concentration of nitrate in groundwater, that have a direct impact on our health when we consume the water, e.g. [21] or that estimate the fluxes of greenhouse gases that are the focus of this paper[2224]. A key underlying issue is how much genomic information is needed to effectively define dominant reactions and the response of a subsurface community to perturbations such as climate change. Initial results suggest that genome-resolved data are needed across a broad range of environments, including those in which organic carbon compounds may be released after being sequestered for millennia. Genome-resolved information will make it possible to capture detailed reaction networks, and ascertain the most important mechanisms controlling the dominant biogeochemical pathways[20]. 
In addition to reshaping our thinking about carbon, nitrogen, hydrogen, and metal cycling in the subsurface, these recent results from genome-resolved studies are providing the basis for incorporating the metabolic capability of the entire subsurface microbial community into predictive models. Successful use of genomic data to inform watershedscale models will greatly improve our ability to understand and manage a wide range of environmental impacts, including how nutrients or water quality within a watershed change in response to droughts, floods and other perturbations that are increasingly attributed to climate change[25] (see the caption for Figure 1 for the definition of the term watershed). The dependence of greenhouse gas fluxes $\left(\mathrm{CO}_{2}, \mathrm{CH}_{4}\right.$, and $\left.\mathrm{N}_{2} \mathrm{O}\right)$ from the shallow subsurface on microbially catalyzed reactions linked to water saturation or fluxes is becoming increasing clear (see for example [26, 27]). At the same time such fluxes are complex both physically and microbiologically with field observations differing from study to study, suggesting that a deeper understanding of microbial processes mediating those fluxes is needed[28].

\section{Novel Microorganisms and Extreme Diversity of Subsurface Microbial Communities}

Several recent research papers report major advances in our understanding of subsurface microbiology. In one such paper[19], researchers reconstructed 8 complete and 789 draft genomes of bacteria from more than 35 phyla, all of which lack isolated representatives and thus are referred to as candidate phyla (CP; phyla are major groups that are as distinct from each other as vertebrates are from mollusks). The organisms from these 35 phyla share features that consistently distinguish them from other bacteria, and group together in what is referred to as the candidate phyla radiation (CPR), a lineage that represents greater than $15 \%$ of the bacterial tree of life. Importantly, bacteria from these phyla mediate hydrogen and organic carbon cycles and appear to depend on other organisms for many basic cellular building materials. However, the diversity of the CPR is remarkable. The first complete genomes from one of the candidate phyla (Peregrinibacteria) differ from most organisms in other lineages of the CPR, in that they have the capacity for nucleotide synthesis, including biosynthesis of cell wall and cell envelope components[29]. However, they also lack of biosynthesis capacities for many required cofactors, fatty acids, and most amino acids, suggesting a symbiotic lifestyle typical of CPR organisms. Further, the CPR organisms generally appear to have different ribosome structures and biogenesis mechanisms than other bacteria. The sequences of the rRNA molecules that form the backbone of the ribosomes of CPR organisms are commonly so unusual that some of these organisms would not be detected in surveys, which routinely use these rRNA sequences[19].

The first extensive genome reconstructions for CPR bacteria indicated that their genomes were very small[30], a feature that suggested that the cell sizes would also be small. This prediction was tested by using cryogenic transmission electron microscopy (cryo-TEM) to study cells that passed through a $0.2 \mu \mathrm{m}$ filter[31]. Such filters are normally used to sterilize water because no cells should be able to pass through them. The cryo-TEM data showed that cells from three phyla (identified by metagenomic analysis of the same samples) have cell sizes of $\sim 0.009 \pm 0.002 \mathrm{~mm}^{3}$ (Figure 3). Characteristics of these cells 
include tightly packed spirals inferred to be DNA, very few densely packed ribosomes, and a variety of cellular appendages that might enable inter-organism interactions.

Another recent study investigated archaea that are present in the subsurface of the same alluvial aquifer and reported findings that largely parallel those for the CRP bacteria[32]. The research significantly expanded our understanding of archaeal diversity and metabolism by genomically sampling a major (but little-studied) superphylum from which no organisms have been isolated. Two new phyla were resolved. The members appear to be organisms with small genomes, possibly nanoarchaea with limited metabolic capacities. The primary biogeochemical impact of these archaea appears to be on anaerobic carbon and hydrogen cycles.

Metagenomics has also been used to explore microbial diversity in aquifer sediments (as opposed to groundwater). Among the most striking findings are the high degree of biological novelty and vast diversity of little known and unknown organisms present[3234]. Recently, the utility of long DNA sequence reads for metagenomic analysis to study such communities was also tested[35]. These newly available, accurate long reads enabled distinction of closely related strains that together comprised a significant fraction of sediment communities and enabled identification of very rare microorganisms. The analysis revealed thousands of species whose abundances were $<0.1 \%$ in all samples. Most of the organisms in this set of rare organisms belong to phyla that are also represented by abundant organisms. Significantly, genes providing the capacity to break down complex organic polymers (glycosyl hydrolases) are more abundant than expected in rare genomes, suggesting that rare species may augment the capability for carbon turnover and confer resilience to changing environmental conditions. Future exploration of community metabolic function and resilience will become increasingly accessible as long-read and nano-pore sequencing are developed, including the likelihood of fielddeployable, next generation sequencing systems[36-39].

\section{Tracking Microbial Shifts in Time and Space}

Another recent paper applied sequencing methods to study the dynamics of subsurface microbial communities. The 133 most abundant organisms in sediment sampled at 5 meters (16 feet) depth from the Rifle aquifer were tracked across 36 different sediment and groundwater environments[40]. Samples were collected centimeters, meters, and tens of meters apart and on seven occasions over 6 years. Analysis of a massive database of DNA sequences (1400 Gb pairs) showed that these 133 organisms were more consistently detected in saturated sediments than in samples from above the water table, from widely separated samples, or from groundwater. Abundance profiles across different locations in the aquifer collected at different sampling times identified consistent organism cohorts. The results suggest that these cohorts are at least partly the result of a shared environmental adaptation. Such research demonstrates that we can track subsurface microbial communities in their environmental context in such a way that the objective of predicting key biogeochemical fluxes in the subsurface (e.g. $\mathrm{CO}_{2}$ or nitrate) is tractable and can be based on the underlying microbially driven processes. This belies the notion that subsurface or even soil microbiology is so heterogeneous and complex 
that it can never be understood and supports the idea that using genomic data to inform watershed-scale models is within reach.

\section{Reaction Pathways, Microbial Diversity, and Functional Resilience}

It has been proposed that metabolic function of microbial communities can be modeled based on 16S rRNA data by assuming functions for detected organisms that are based on inferences from available database genomes. Such an approach is clearly inapplicable for subsurface studies, where reference genomes are not available (except from metagenomics) and many organisms (in fact, members of entire phyla) escape detection by rRNA sequencing approaches. Thus, we argue that the level of insight provided by genome-resolved studies is critical to understanding functionality of subsurface systems. A subset of the complex microbial reaction pathways might be expected $a$ priori even in the absence of deep metagenomic analysis. For example, reactive transport models can be based on reaction networks inferred from 16S rRNA gene surveys and associated geochemical dynamics of the system [41-43]). Such a model could just incorporate the pathways as individual reaction networks without the need for extensive metagenomic characterization. What limits such an approach is the fact that the unit catalyzing biogeochemical transformations is the individual microbial cell. Specific microbial functions are combined within cells, which operate in their microbial community context. An understanding of which capacities co-occur and which resources are exchanged among species is critical to understanding the rate-limiting steps in reaction networks. Given the massive genomic information for sites like the Rifle alluvial aquifer, the genetic capacity and the relative numbers of microorganisms in aquifers can likely be used to infer what underlying metabolic processes are needed to drive the carbon, nitrogen, sulfur and hydrogen cycles[33]. Detailed evidence for such metabolic processes could reshape the way we construct biogeochemical reactive transport models.

Another facet of detailed metagenomic information is the ability to assess metabolic 'resiliency' within a given system. The 'microbial seed bank' confers an advantage in the face of climate-induced changes in soil/sediment temperature, moisture content, redox conditions, salinity, etc. Understanding of resiliency requires information about very low abundance organisms and is critical to predicting trajectories of disturbed ecosystems. For example, Geobacter sp. may be present at very low abundance levels in sediment, yet relaxation of organic carbon limitation by acetate addition causes this organism to 'bloom' and dominate the active microbial community[44]. Proliferation of Geobacter species that also can fix $\mathrm{N}_{2}$ into a form bioavailable to the larger community has broader implications for the overall ecosystem function[45, 46].

It is our thesis, then, that given deep genome-resolved information, we should be able to make robust predictions about how watershed systems, including soils and variably saturated underlying sediments, will respond to environmental changes. Such shifts could be in the form of changes in rainfall (soil moisture and water table excursions) or in vegetation (microbe-plant/root associations). Such shifts likely will have broad, aggregated impacts on cycling of nutrients such as C, N, S, and P [47]. Limited progress is being made toward models that include genomic diversity in prediction of microbial community response to changing environmental conditions[20]. An example of an 
ecosystem impact arising from changing microbial community composition might be the proliferation of chemolithoautotrophs in response to subsurface nitrate amendment[48], accompanied by increased conversion of $\mathrm{CO}_{2}$ into biomass. Other examples include release of metal contaminations adsorbed to ferric iron oxyhydroxide mineral surfaces following increased $\mathrm{Fe}$ reduction due to stimulation of Geobacter sp., by changes in organic carbon availability[41, 44], and an increase in U(VI) reduction rate in response to amendment with bicarbonate and acetate compared to acetate alone[41]. Ultimately, genomes of the microbial community members encode the capacities that determine the forms of such responses. Looking forward, the challenge will be to validate the consistency and predictability of such responses by experiments and observations that combine deep metagenomics and transcriptomics with detailed biogechemical and isotopic observations capable of revealing the details of reactions that modify key biogeochemical fluxes.

\section{Implications for Subsurface Biogeochemical Processes Including Greenhouse Gas Fluxes from the Subsurface}

Coming into sharper focus is the vast diversity of microorganisms that mediate critically important biogeochemical reactions[29]. In Figure 4 (Key Figure) we illustrate the principal capacities predicted from genomic information. Each organism that carries out such processes does so at a rate determined by other aspects of its physiology in concert with the other transformations that it is equipped to mediate. Many of these microbially controlled reactions impact the fluxes of the greenhouse gases $\mathrm{CO}_{2}, \mathrm{CH}_{4}$, and $\mathrm{N}_{2} \mathrm{O}$ through water bodies and into the atmosphere[26] (Figure 4). Of course, no single organism will have all of the catalytic machinery for all of the reactions shown. At one end member are the CPR bacteria, which likely cannot to survive on their own but rather depend on other community members for critical resources, yet provide metabolically valuable fermentation products. At another end are the Zixibacteria[35, 47], which have a broad metabolic repertoire that probably explains their dominance in the timevarying environment close to the fluctuating boundary between the water table and vadose zone. Also important but not depicted in Figure 4 is predation by viral and protist populations [49-56]. While individual microbial species may produce a constituent of interest (e.g., methane from Candidatus 'Methanoflorens stordalenmirensis' or Methanosarcina in thawing permafrost $[5,26,57,58]$ ), other community members (possibly CPR that generate $\mathrm{H}_{2}$ ) may be required to support $\mathrm{CH}_{4}$ production. This is likely emblematic of subsurface ecosystem function: microorganisms growing in community context drive biogeochemical processes. Thus, understanding the diversity and capacities of these organisms will be critical for predicting greenhouse gas fluxes to and from the subsurface.

\section{Concluding Remarks}

The microbiology of the shallow terrestrial subsurface plays a key role in organic carbon and hydrogen cycles, ultimately contributing to greenhouse gas fluxes to and from the subsurface. Previously little known and unknown archaeal and bacterial species contribute to a variety of interlinked chemical transformations in the nitrogen and sulfur cycles, as well as carbon and hydrogen cycles. But how do these communities change 
with time, and what are the master controls that influence the microbial community makeup? Or, conversely, what biogeochemical or physical features does that the microbial community modify as a result of its underlying emergent properties? While models of microbial community interactions are in development, we do not as yet have the ability to make de novo predictions about the response of subsurface microbial communities to perturbations. We argue that a deeper, mechanistic level of understanding of microbial species interactions, competition, and function are required to effectively model subsurface greenhouse gas fluxes at the watershed scale, particularly under the ongoing perturbation of climate change. To address this gap, future research needs to systematically interrogate genomics of representative subsurface biomes, coupled with observations of subsurface genomic, transcriptomic, and proteomic responses to both short-term (human induced, including laboratory scale) and long-term perturbations (see Outstanding Questions). Such research is consistent with the recently announced microbiomes initiative $[7,8]$. Our ability in the future to match both aggregate fluxes and detailed microbial observations of perturbations with models will serve as the test of our modeling capabilities. Further, these results will provide a direct measure of the extent to which mechanistic microbial genomics are needed to sustain model fidelity.

\section{Acknowledgements}

This research was supported by the US Department of Energy (DOE), Office of Science, Office of Biological and Environmental Research, Subsurface Biogeochemistry Research Program under award number DE-AC02-05CH11231 to Lawrence Berkley National Laboratory (Genomes to Watershed Scientific Focus Area) and award number DESC0004918 (Systems Biology Knowledge Base Focus Area). Lawrence Berkeley National Laboratory is operated by the University of California for the US DOE. Genomic sequencing was performed at the US DOE Joint Genome Institute, a DOE Office of Science User Facility, supported under contract DE-AC02-05CH11231. Transcriptomics work was conducted at the Environmental Molecular Sciences Laboratory, a National Scientific User Facility sponsored by the DOE Office of Biological and Environmental Research and located at Pacific Northwest National Laboratory (PNNL). PNNL is operated by Battelle for the US DOE under contract AC0676RLO 1830. 


\section{Figure Legends}

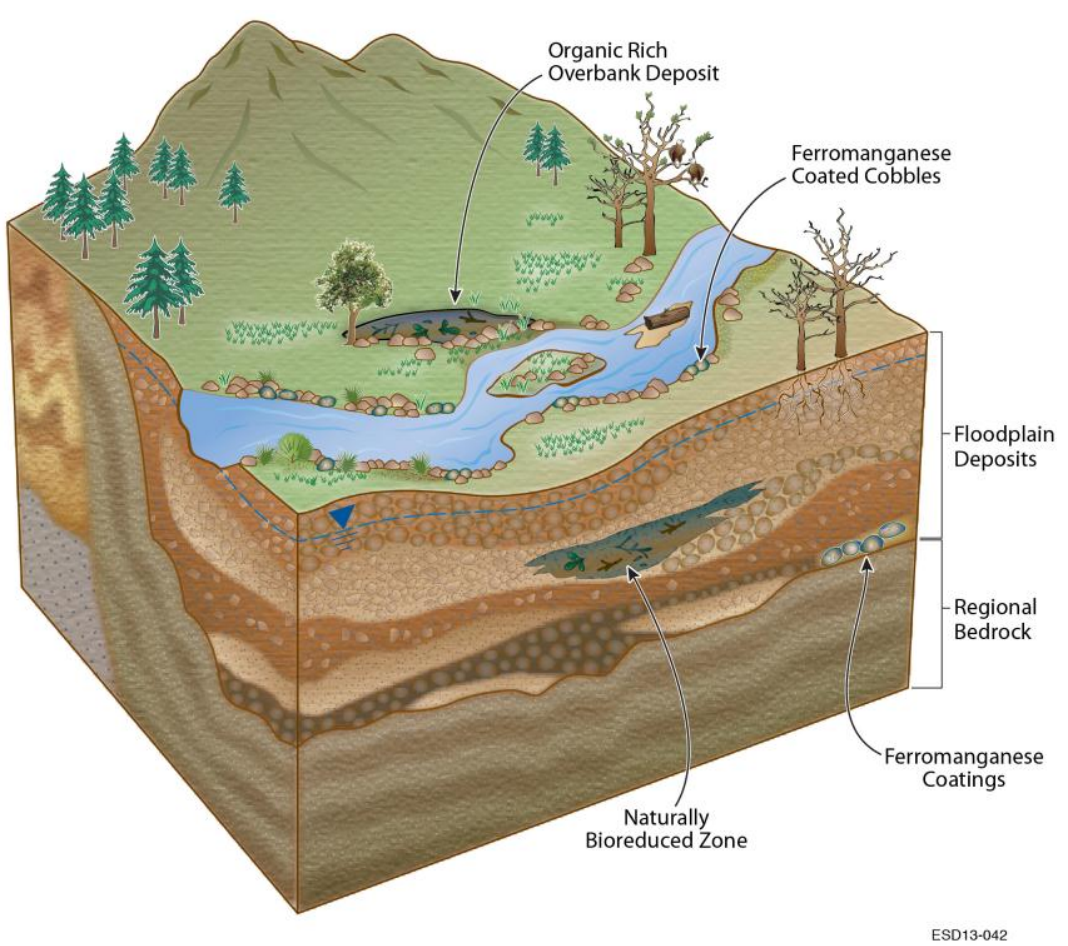

Figure 1. Illustration Showing Organic Carbon Deposits and Naturally Bioreduced Zones. These are created when organic matter accumulations, typical of most floodplains, are buried in the shallow subsurface $(<10 \mathrm{~m}$ below the terrestrial surface of the earth). Genomic information on microorganisms in such zones provides information about their metabolic capacities enabling modeling of subsurface biogeochemical processes. This will allow accurate prediction of how subsurface processes impact quality of water and nutrient cycling within watersheds as well as the flux of greenhouse gases to the atmosphere. The term watershed here refers to a region or area of the terrestrial surface that is bounded by a divide and drains to a particular river or water body, including all the streams or rivers that drain into it. Watersheds are a key functional unit of the earth governing water resources, and mediating biogeochemical processes that support all terrestrial life. For details of the microbially catalyzed biogeochemical reactions that occur in the subsurface, see Figure 4. 


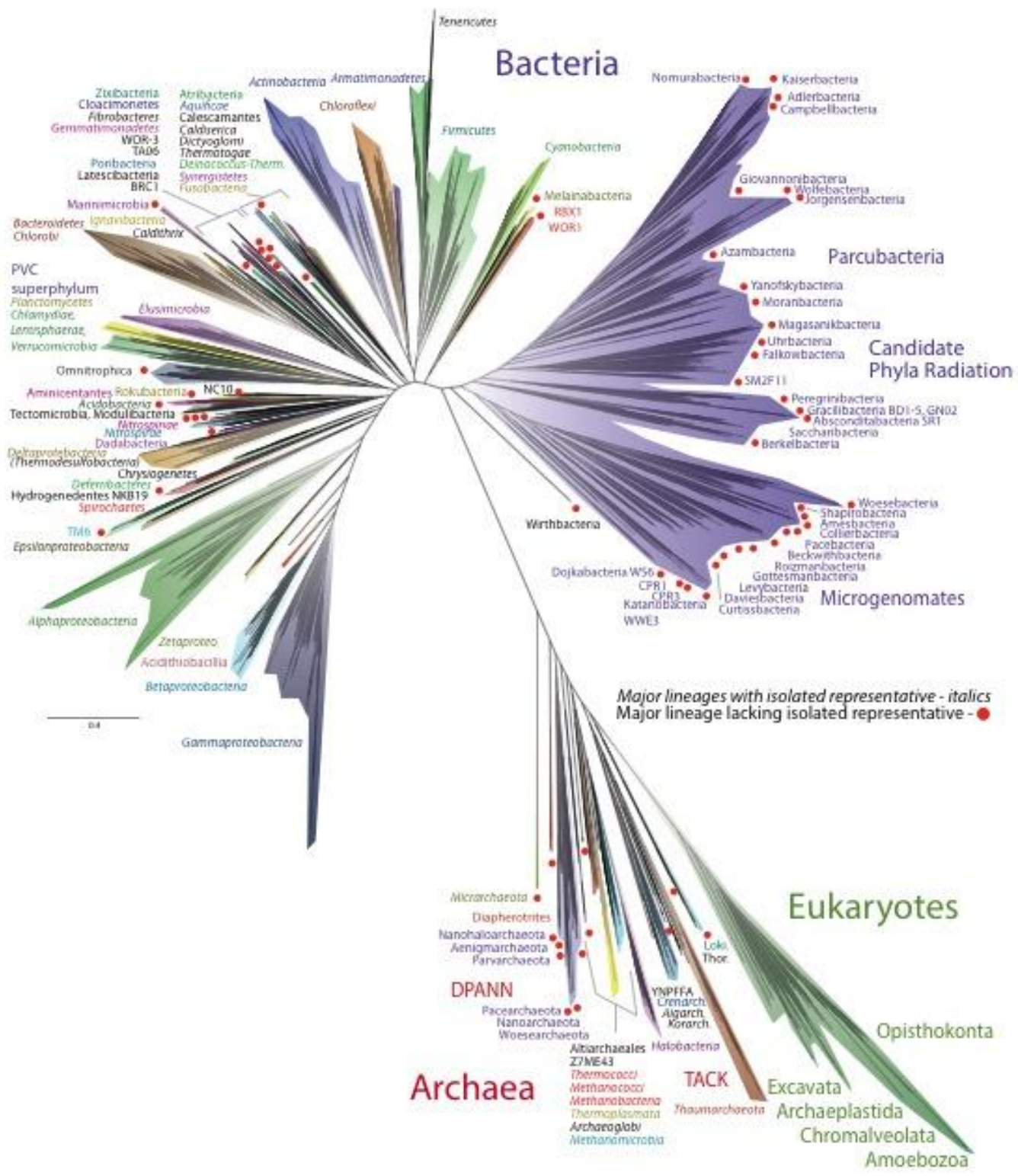

Figure 2. Tree of Life Generated by Hug and Colleagues in 2016[9]. The total diversity represented by available sequenced genomes including 100 bacterial phyla, 17 archaeal phyla, and 5 eukaryotic super-groups. Colors and names represent major lineages. Well-characterized lineages are in italic font, whereas lineages lacking a cultured representative are highlighted in non-italic font and with red circles. DPANN and CPR are assigned single colors as they are without isolated representatives. See [9] for additional information. This figure is reproduced 
unaltered from [9] under a Creative Commons CC BY license with permission from Nature Microbiology.
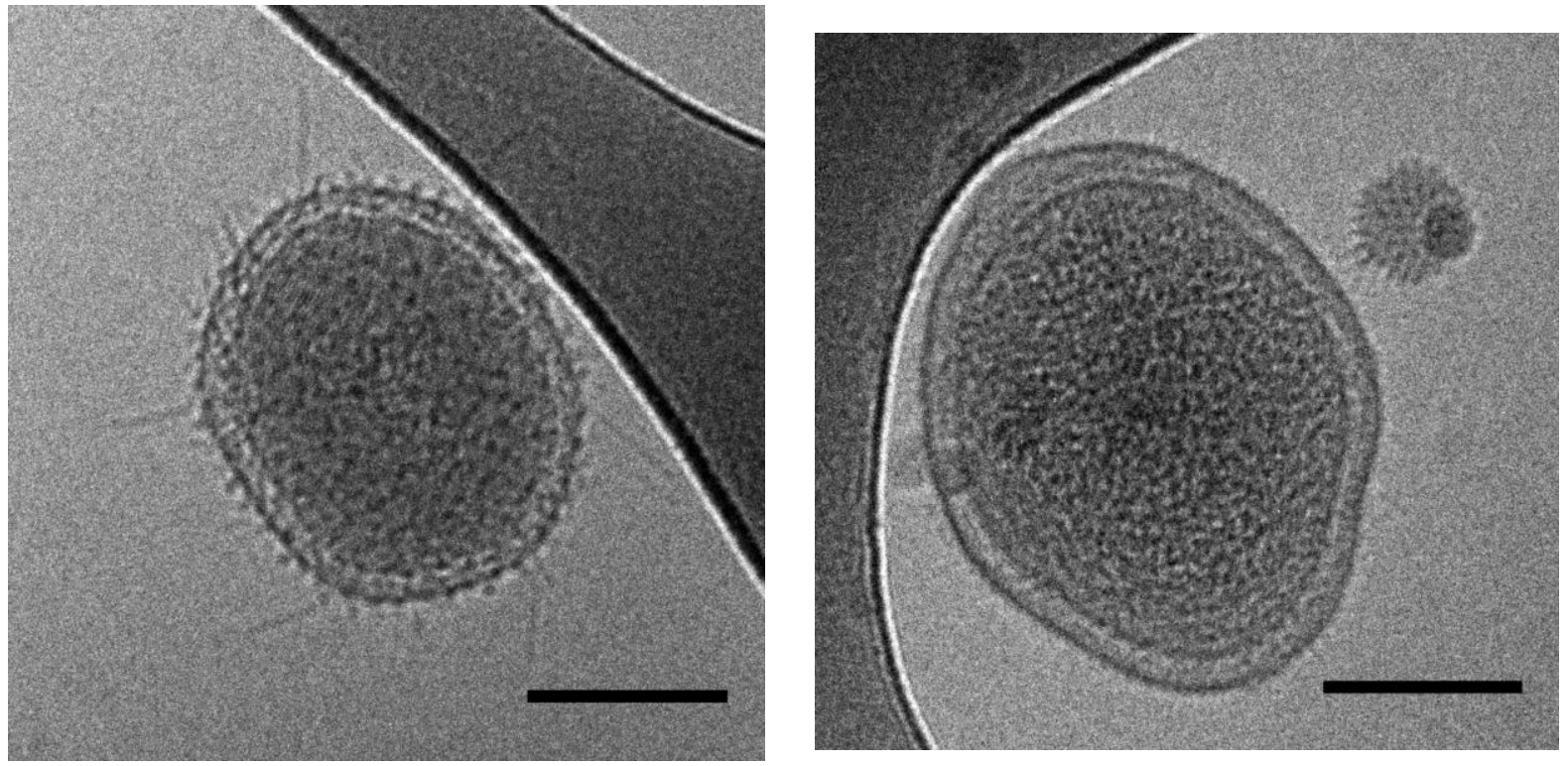

Figure 3. Cryo-TEM Images Showing the Morphology, Size and Some Key Features of UltraSmall Bacteria. The cell envelope includes distinct outer layer (S-layer). Pili-like structures are clearly visible in the image on the left and in some cases these pili apparently connect to adjacent bacteria. Scale bar is 100 nanometers or about $1 / 500^{\text {th }}$ to $1 / 1,000^{\text {th }}$ of the diameter of a human hair. Note that these bacteria are so small that apparent low resolution of the images reflects the resolution limits of the cryo-TEM technique. See also Figures 3 and 4 in [31]. 


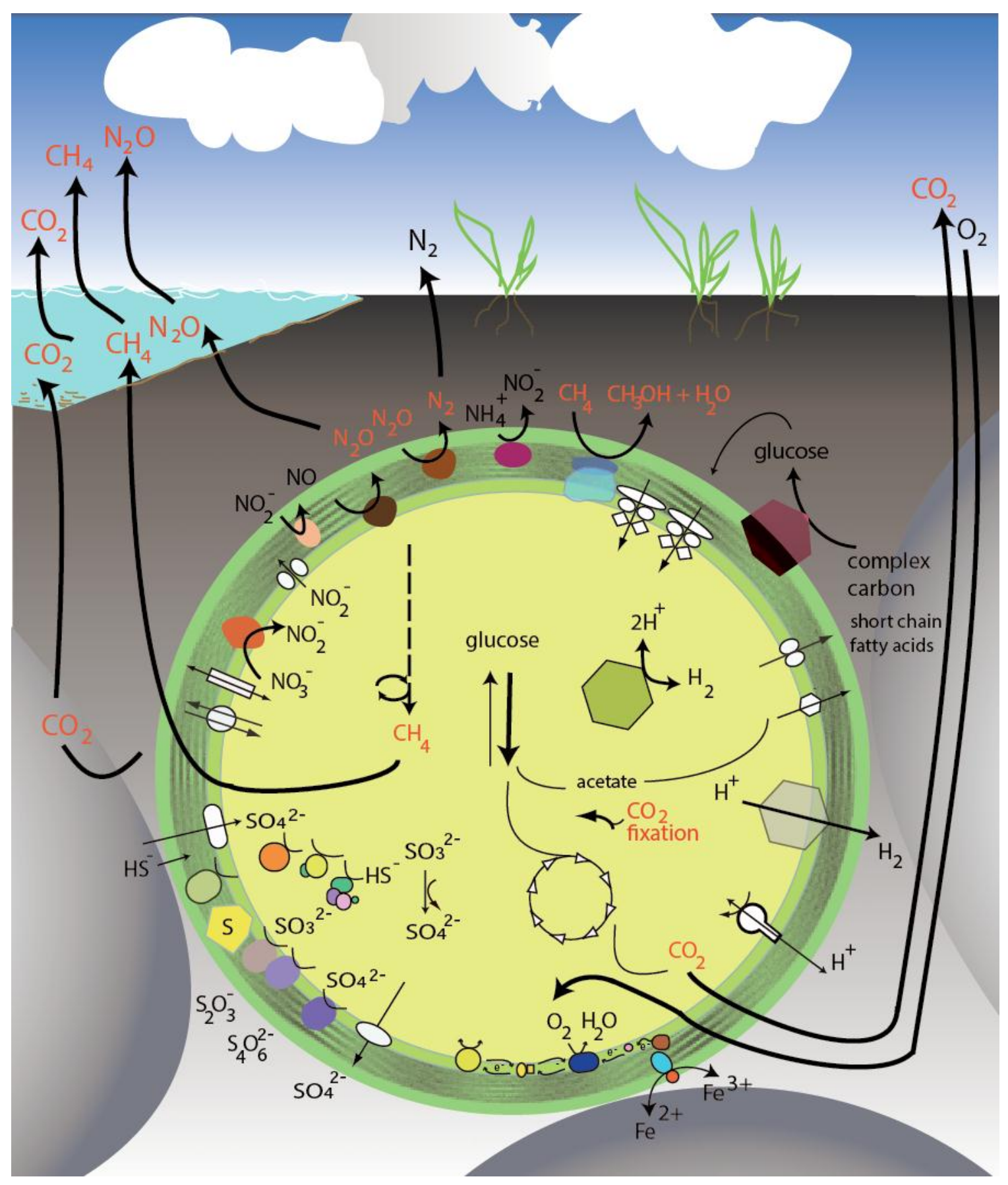

Figure 4. Diagram Illustrating Microbially Mediated Reactions Occurring in the Shallow Terrestrial Subsurface. This figure highlights information obtained from genome-resolved studies that inform our understanding of these microbially mediated reactions. The red text highlights pathways that impact atmospheric greenhouse gas concentrations. Only rarely would any individual cell be expected to have all of the capacities shown in this schematic. 


\section{References Cited}

1 Raymond, J. and Alsop, E.B. (2015) Microbial evolution in extreme environments: microbial migration, genomic highways, and geochemical barriers in hydrothermal ecosystems. Environmental Systems Research 4, 1-12

2 Kieft, T., et al. (2015) Workshop to develop deep-life continental scientific drilling projects. Sci Dril 19, 43-53

3 Emerson, J.B., et al. (2015) Metagenomic analysis of a high carbon dioxide subsurface microbial community populated by chemolithoautotrophs and bacteria and archaea from candidate phyla. Environ Microbiol 10.1111/1462-2920.12817 4 Tan, J., et al. (2015) Unraveling interactions in microbial communities - from cocultures to microbiomes. J Microbiol. 53, 295-305

5 Hultman, J., et al. (2015) Multi-omics of permafrost, active layer and thermokarst bog soil microbiomes. Nature 521, 208-212

6 Herbst, F.-A., et al. (2016) Enhancing metaproteomics-The value of models and defined environmental microbial systems. PROTEOMICS 16, 783-798

7 Alivisatos, A.P., et al. (2015) A unified initiative to harness Earth's microbiomes. Science 350, 507-508

8 Dubilier, N., et al. (2015) Microbiology: Create a global microbiome effort. Nature 526,631

9 Hug, L.A., et al. (2016) A New View of the Tree of Life. Nature Microbiology doi:10.1038/nmicrobiol.2016.48

10 Abram, F. (2015) Systems-based approaches to unravel multi-species microbial community functioning. Computational and structural biotechnology journal 13, 2432

11 Woese, C.R., et al. (1990) Towards a natural system of organisms: proposal for the domains Archaea, Bacteria, and Eucarya. Proceedings of the National Academy of Sciences 87, 4576-4579

12 Reed, D.C., et al. (2015) Predicting the response of the deep-ocean microbiome to geochemical perturbations by hydrothermal vents. The ISME journal 9, 1857-1869

13 Osburn, M.R., et al. (2014) Chemolithotrophy in the continental deep subsurface: Sanford Underground Research Facility (SURF), USA. Frontiers in microbiology 5, 10.3389/fmicb.2014.00610

14 Lynch, M.D.J. and Neufeld, J.D. (2015) Ecology and exploration of the rare biosphere. Nat Rev Micro 13, 217-229

15 Konstantinidis, K.T. and Rosselló-Móra, R. (2015) Classifying the uncultivated microbial majority: A place for metagenomic data in the Candidatus proposal. Systematic and applied microbiology 38, 223-230

16 Stefani, F.O.P., et al. (2015) Culture-Dependent and -Independent Methods Capture Different Microbial Community Fractions in Hydrocarbon-Contaminated Soils. PLOS ONE 10, e0128272

17 Garza, D.R. and Dutilh, B.E. (2015) From cultured to uncultured genome sequences: metagenomics and modeling microbial ecosystems. Cellular and Molecular Life Sciences, 1-22 
18 Zhou, J., et al. (2015) High-Throughput Metagenomic Technologies for Complex Microbial Community Analysis: Open and Closed Formats. mBio 6, e02288-02214 19 Brown, C.T., et al. (2015) Unusual biology across a group comprising more than $15 \%$ of domain Bacteria. Nature 523, 208-211

20 Reed, D.C., et al. (2014) Gene-centric approach to integrating environmental genomics and biogeochemical models. Proceedings of the National Academy of Sciences 111, 1879-1884

21 Arora, B., et al. (2016) Influence of Hydrological, Biogeochemical and Temperature Transients on Subsurface Carbon Fluxes in a Flood Plain Environment. Biogeochemistry 127, 367-396

22 Jarecke, K.M., et al. (2016) Coupled soil oxygen and greenhouse gas dynamics under variable hydrology. Soil Biology and Biochemistry 95, 164-172

23 Blagodatsky, S. and Smith, P. (2012) Soil physics meets soil biology: towards better mechanistic prediction of greenhouse gas emissions from soil. Soil Biology and Biochemistry 47, 78-92

24 Singh, B.K., et al. (2010) Microorganisms and climate change: terrestrial feedbacks and mitigation options. Nature Reviews Microbiology 8, 779-790 25 Diffenbaugh, N.S., et al. (2015) Anthropogenic warming has increased drought risk in California. Proceedings of the National Academy of Sciences 112, 3931-3936 26 McCalley, C.K., et al. (2014) Methane dynamics regulated by microbial community response to permafrost thaw. Nature 514, 478-481

27 Lagomarsino, A., et al. (2016) Past water management affected GHG production and microbial community pattern in Italian rice paddy soils. Soil Biology and Biochemistry 93, 17-27

28 Hall, S.J., et al. (2015) Environmental forcing does not induce diel or synoptic variation in the carbon isotope content of forest soil respiration. Biogeosciences 12, 5143-5160

29 Anantharamana, K., et al. (2016) Analysis of five complete genome sequences for members of the class Peribacteria in the recently recognized Peregrinibacteria bacterial phylum. PeerJ 4, e1607 30 Wrighton, K.C., et al. (2012) Fermentation, Hydrogen and Sulfur Metabolism in Multiple Uncultivated Bacterial Phyla. Science 337, 1661-1665

31 Luef, B., et al. (2015) Diverse uncultivated ultra-small bacterial cells in groundwater. Nature communications 6, 10.1038/ncomms7372

32 Castelle, C.J., et al. (2015) Genomic expansion of domain archaea highlights roles for organisms from new phyla in anaerobic carbon cycling. Current Biology 25, 690701

33 Hug, L.A., et al. (2015) Critical biogeochemical functions in the subsurface are associated with bacteria from new phyla and little studied lineages. Environ Microbiol 10.1111/1462-2920.12930

34 Evans, P.N., et al. (2015) Methane metabolism in the archaeal phylum Bathyarchaeota revealed by genome-centric metagenomics. Science 350, 434-438 35 Sharon, I., et al. (2015) Accurate, multi-kb reads resolve complex populations and detect rare microorganisms. Genome research 25, 534-543

36 Feng, Y., et al. (2015) Nanopore-based Fourth-generation DNA Sequencing Technology. Genomics, Proteomics \& Bioinformatics 13, 4-16 
37 Pennisi, E. (2016) Pocket DNA sequencers make real-time diagnostics a reality. Science 351, 800-801

38 Lindsay, S. (2016) The promises and challenges of solid-state sequencing. Nature nanotechnology 11, 109-111

39 Gasc, C., et al. (2015) Capturing prokaryotic dark matter genomes. Research in Microbiology 166, 814-830

40 Hug, L.A., et al. (2015) Aquifer environment selects for microbial species cohorts in sediment and groundwater. The ISME journal 9, 1846-1856

41 Long, P.E., et al. (2015) Bicarbonate impact on U(VI) bioreduction in a shallow alluvial aquifer. Geochimica Et Cosmochimica Acta 150, 106-124

42 Yabusaki, S., et al. (2012) Coupled-Process Modeling of a Uranium

Bioremediation Field Experiment (Abstract). Mineralogical Magazine 76, 2562

43 Bao, C., et al. (2014) Uranium Bioreduction Rates Across Scales: Biogeochemical "Hot Moments" and "Hot Spots" During a Field Biostimulation Experiment at Rifle, Colorado. Environ. Sci. Technol. 48, 10116-10127

44 Anderson, R.T., et al. (2003) Stimulating the in situ activity of Geobacter species to remove uranium from the groundwater of a uranium-contaminated aquifer. Appl Environ Microb 69, 5884-5891

45 Mouser, P.J., et al. (2009) Influence of heterogeneous ammonium availability on bacterial community structure and the expression of nitrogen fixation and ammonium transporter genes during in situ bioremediation of uraniumcontaminated groundwater. Environ Sci Technol 43, 4386-4392

46 Bazylinski, D.A., et al. (2000) N2 - dependent growth and nitrogenase activity in the metal - metabolizing bacteria, Geobacter and Magnetospirillum species. Environ Microbiol 2, 266-273

47 Baker, B.J., et al. (2015) Genomic resolution of linkages in carbon, nitrogen, and sulfur cycling among widespread estuary sediment bacteria. Microbiome 3, 14 48 Jewell, T.N.M., et al. (2016) Metatranscriptomic evidence of pervasive and diverse chemolithoautotrophy relevant to $\mathrm{C}, \mathrm{S}, \mathrm{N}$, and Fe cycling in a shallow alluvial aquifer. ISME J 10.1038/ismej.2016.1025

49 Worden, A.Z., et al. (2015) Rethinking the marine carbon cycle: Factoring in the multifarious lifestyles of microbes. Science 347, 1257594

50 Roux, S., et al. (2015) Viral dark matter and virus-host interactions resolved from publicly available microbial genomes. eLife 4, e08490

51 Holmes, D.E., et al. (2013) Enrichment of specific protozoan populations during in situ bioremediation of uranium-contaminated groundwater. ISME J 7, 1286-1298 52 Engelhardt, T., et al. (2014) High virus-to-cell ratios indicate ongoing production of viruses in deep subsurface sediments. The ISME journal 8, 1503-1509

53 Pedersen, K. (2012) Subterranean microbial populations metabolize hydrogen and acetate under in situ conditions in granitic groundwater at $450 \mathrm{~m}$ depth in the Äspö Hard Rock Laboratory, Sweden. FEMS microbiology ecology 81, 217-229

54 Legendre, M., et al. (2015) In-depth study of Mollivirus sibericum, a new 30,000$\mathrm{y}$-old giant virus infecting Acanthamoeba. Proceedings of the National Academy of Sciences 112, E5327-E5335 
55 Holmes, D.E., et al. (2015) Evidence of Geobacter-associated phage in a uraniumcontaminated aquifer. The ISME journal 9, 333-346

56 Holmes, D.E., et al. (2014) Methane production from protozoan endosymbionts following stimulation of microbial metabolism within subsurface sediments.

Frontiers in microbiology 5, 366

57 Mondav, R., et al. (2014) Discovery of a novel methanogen in thawing permafrost. Nature Communications 5, 3212

58 Jansson, J.K. and Taș, N. (2014) The microbial ecology of permafrost. Nature

Reviews Microbiology 12, 414-425 